\title{
A Comparison of Digital Length Estimators for Image Features
}

\author{
V. Toh ${ }^{1}$, C.A. Glasbey ${ }^{2}$, and A.J. Gray ${ }^{1}$ \\ 1 Department of Statistics and Modelling Science, University of Strathclyde, \\ Glasgow, G1 1XH, UK \\ vivian@stams.strath.ac.uk, alison@stams.strath.ac.uk \\ 2 Biomathematics and Statistics Scotland, King's Buildings, Edinburgh EH9 3JZ, UK \\ chris@bioss.ac.uk
}

\begin{abstract}
Image analysis methods for estimating size of object features extract pixel-based measurements, after object segmentation, then convert these to an estimate of actual size; e.g. segmentation of a cell in a randomly located 2-D cross-sectional image, counting no. of pixels on the cell boundary, and converting to an estimate of cell surface area using geometrical formulae. Stereology takes a quite different approach to estimating higher dimensional properties of an object, by using a randomly orientated 2-D specimen section or 2-D projection of a 3-D object. Geometrical properties and sampling theory enable inference of 3-D properties; e.g. feature length is estimated by counting intersections with a randomly superimposed test grid with fixed known spacing. This work compares these two approaches for image feature length estimation, using a simulation study. We generate binary straight line structures and planar curves of known size and compare results from several different estimators of feature length, including a novel estimator which weights pixel count by estimating local curve orientation.
\end{abstract}

\section{Motivation}

A main aim of image analysis is to extract quantitative information, such as feature size. Often this involves curve length estimation, e.g. length of fungal hyphae viewed under a microscope, or perimeter length of an object in an image, or estimation of area. For example in Figure 1(a), a microscope mycelial image, simple measures such as branch lengths and overall structure size are of interest as well as spatial distribution of the fungus. Equally, three-dimensional properties may be of interest, such as object volume or surface area, either inferred from two-dimensional measurements, or estimated directly. The microscope cell image in Figure 1(b) shows a stained histological section of mouse mammary tissue, representing proliferating cells (smaller blue cells), apoptotic cells (larger brown cells), milk sacs (lightest, irregular areas) and other types of tissue (darker background). As well as cell counts, aspects of interest to the experimental scientists include estimation of the surface area and volume of the milk sacs from such two-dimensional images. 
For real images true feature size is not known and the most feasible means of comparing possible estimators is via a simulation study, which allows assessment of the error of estimation since true feature length is known. In this paper, we compare estimators of feature length, using a small-scale simulation-based comparison of pixel-based methods, both well-known estimators and some simple variations on these, described in Section 2. We also consider stereological methods as they offer an alternative statistical approach to feature measurement which has the advantage of allowing direct estimation of three-dimensional properties. Stereological estimation is described in Section 3. Section 4 describes the simulation study, Section 5 shows some results and Section 6 presents some conclusions.

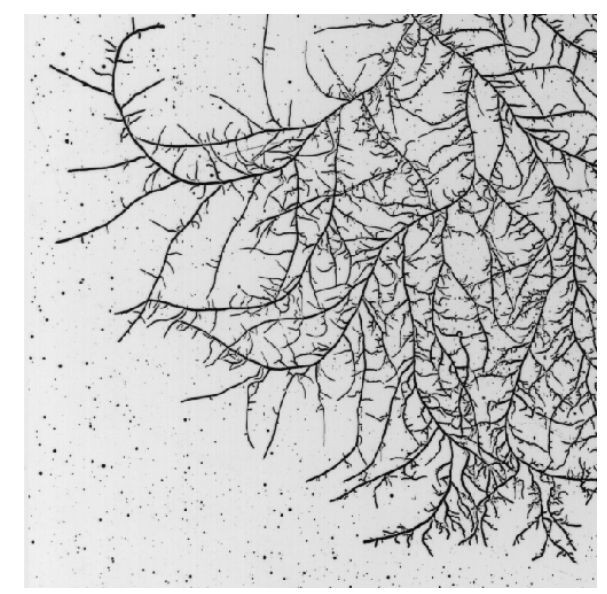

(a)

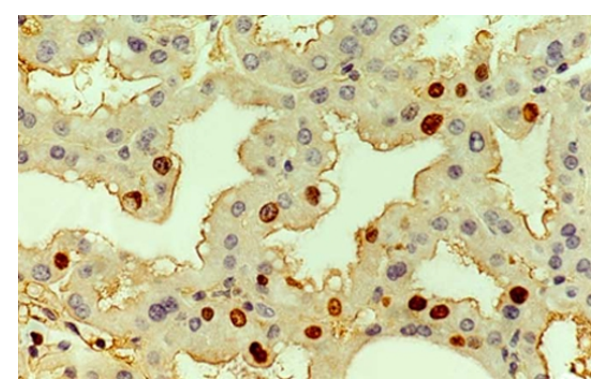

(b)

Fig. 1. (a) A grey-scale mycelial image; (b) A stained colour cell image of mouse mammary tissue

\section{Image Analysis Approach}

In practice an image is usually represented in digitized form. Most length estimators are based on either pixel counts or properties of the chain-code representation of a digital line or curve.

\subsection{Pixel-based Length Estimators}

A rough estimate of length simply counts no. of pixels. In general this is not unbiased for length of the underlying smooth curve, and pixel count differs for 8-connected and 4-connected lines. Glasbey and Horgan [6], Ch. 6, show that, if 
all orientations of a line are equally likely, length is estimated unbiasedly by $\frac{N 8}{0.900}$ or $\frac{N 4}{1.273}$, where N8 and N4 are no. of pixels in an 8-connected and 4-connected line respectively. A third simple estimator measures distance between diagonally adjacent pixels as $\sqrt{2}$. The no. of diagonal links is $N 4-N 8$, the remaining $N 8-(N 4-N 8)$ links in an 8-connected boundary are one pixel unit long, so total length is $N 8-(N 4-N 8)+\sqrt{2}(N 4-N 8)=0.414 N 4+0.586 N 8$. For equally likely orientations, expected number of diagonally adjacent pixels is 1.055, which could again be used as a correcting factor. This third estimator has lower variance.

\subsection{Chain-code Estimators}

A digitized representation of a continuous line is often stored compactly as a chain-code string. Different continuous lines may have the same digitized representation and hence the same chain-code. From an initial pixel location, the digital line is represented as a series of moves in different directions. A chaincode labels the links between every pair of pixels (see Fig. 2). An 8-connected chain-code string consists of even chain-code elements (horizontal and vertical links) and odd elements (diagonal links). Corner counts are movements from odd to even or even to odd chain-codes (Fig. 2 (c)). Different chain-codes can have the same no. of even, odd and corner chain codes, denoted as $n_{e}, n_{o}$ and $n_{c}$ respectively, and hence the same value of a length estimator.

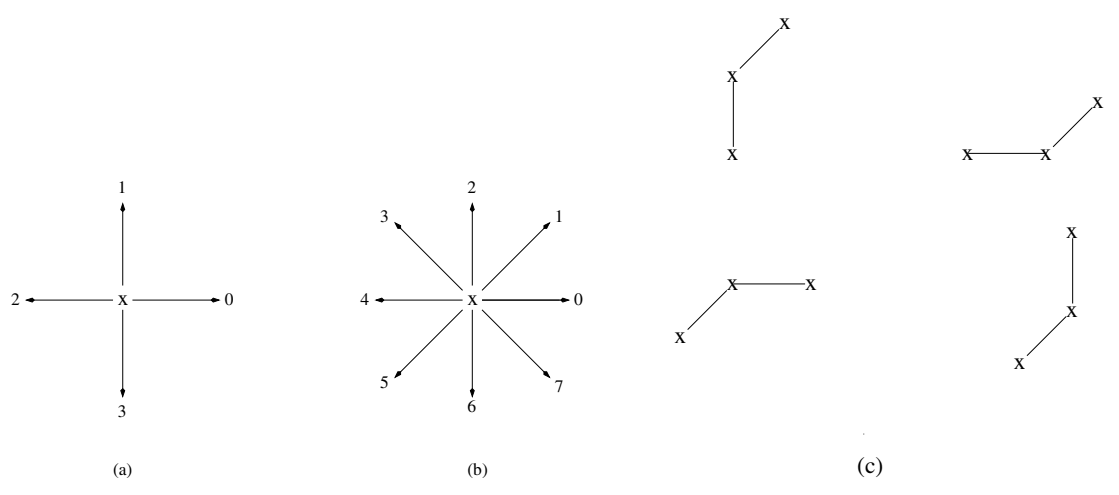

Fig. 2. (a) Chain-codes for moves in a 4-connected neighbourhood, from an initial pixel $\mathrm{x}$; (b) The Freeman [5] chain-code scheme for the 8-connected case; (c) Possible corner count moves for a digitized line with slope in the first quadrant. We count no. of times a horizontal/vertical link is followed by a diagonal one, or vice versa

Table 1 summarises various estimators which are a linear combination of these parameters, i.e. $L=a n_{e}+b n_{o}+c n_{c}$. The simplest is total no. of chaincode elements (links) $n$ along an 8-connected contour, though this is biased for long straight line segments (Dorst and Smeulders [3]). Dividing by 0.900 makes 
Table 1. Summary of various estimators examined

\begin{tabular}{|c|c|c|c|c|}
\hline \multirow[b]{2}{*}{ Estimator } & \multicolumn{3}{|c|}{ Coefficients } & $\begin{array}{l}\text { Literature RDEV } \\
\qquad(\times 100)\end{array}$ \\
\hline & & & & \\
\hline links & 1 & 1 & 0 & 16 \\
\hline$\frac{\text { links }}{0.900}$ & 1.1107 & 1.1107 & 0 & 11 \\
\hline Freeman & 1 & $\sqrt{2}=1.414$ & 0 & 6.6 \\
\hline Kulpa & 0.9481 & 1.343 & 0 & 2.6 \\
\hline Corner count & 0.980 & 1.406 & -0.091 & 0.8 \\
\hline
\end{tabular}

it unbiased and approximately equal to $\frac{N 8}{0.900}$. Freeman [4] counted even links as length 1 and odd ones as length $\sqrt{2}$, to give exact length of the digital arc. This is biased (too large), though commonly used. To reduce bias, Kulpa [8] found the coefficients to minimise expected error for long line segments. If corners are treated distinctly, the corner count estimator (Vossepoel and Smeulders [9]) minimises mean square error for all long straight strings (with $\mathrm{n}=1000$ ), and is asymptotically unbiased. It has the lowest asymptotic root mean square deviation (RDEV) of these estimators.

\section{Stereological Approach}

Stereological methods mathematically relate $3-\mathrm{D}$ parameters defining a structure (such as rock, metals or living tissues) to lower dimensional measurements from sections through the structure. For example, microscope slides of thin slices through tissue may contain information about number of cells, capillary length, area of membranes, or volume of different tissue types. Simple stereological relationships between these lower and higher dimensional quantities, when used with suitable sampling techniques, give very efficient and accurate measurements. Probes (points, lines, planes or volumes) are placed randomly on the sectioned feature and intersections counted. Random sampling ensures unbiased estimation (Howard and Reed [7]).

To estimate planar curve length this approach counts intersections between the curve and a line grid (Buffon [2]; Baddeley [1]). Consider a needle of length $l$ tossed 'at random' onto a floor of planks separated by straight line edges a constant perpendicular distance h apart, where $h \geq l$ (Fig. 3). Needle position is given by the coordinates (x,y) of a reference point on the needle (one end, say), and the orientation angle $\theta$ of the needle. Buffon [2] showed that the probability $p$ of crossing the planks is $\frac{2 l}{h \pi}$, if all directions $\theta$ are equally likely. 

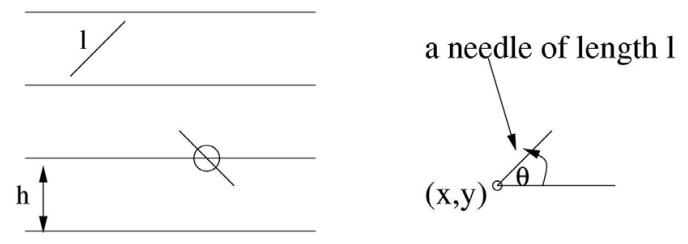

Fig. 3. A needle of length $l$ with reference point $(x, y)$ is dropped at random orientation $\theta$ onto a grid of equal spacing h; it may or may not intersect an edge or grid line

If an irregular planar curve of length $l$ is placed at random many times on the same equally spaced lines, the average no. of intersections, $\bar{N}$, can be obtained as follows. Divide the curve into nearly straight short segments of length $\Delta l$ $(\Delta l \leq h)$. Each segment takes random position as the curve as a whole does, so the probability that any one segment intersects the array is $p_{1}=\frac{2 \Delta l}{h \pi}$. Hence the expected no. of intersections is $\bar{N}=p_{1} \cdot \frac{l}{\Delta l}=\frac{2 l}{h \pi}$, so $l=\bar{N} h \frac{\pi}{2}$, and $\hat{l}=N h \frac{\pi}{2}$ from a single random placement of the curve gives an unbiased estimator of $l$ (Howard and Reed [7], pp. 35-36). In practice the spacing $\mathrm{h}$ is taken such that $h<l$, where $l$ is length of the complete line or curve rather than length of a sub-segment or needle (which can be arbitrarily short). Complete coincidence of a straight line feature with a grid line is defined to give one intersection.

\section{Simulation Study}

We consider both straight lines and planar curves. Straight line features are generated with start point $(1,1)$ at random angle $\theta \in[0, \pi / 2]$ to the horizontal. The line is taken to extend the length of the image plane and a digitized 8-connected version produced. The true length of the underlying smooth line is calculated using Euclidean distance between the (real) end points. We also generate random quartic polynomial curves. The turning points and endpoints are generated randomly in the continuous plane and the coefficients of the interpolating quartic are found. A digitised 8-connected version is then generated. True curve length is computed using numerical approximation of arc length.

The true lengths are used to assess estimator performance over 100 simulations for various sizes of image. For each estimator, the following performance measures were used: Minimum, Maximum and Median Absolute Deviation (Min $\mathrm{AD}$, Max $\mathrm{AD}$, and MAD), as well as number of times the estimator is larger (NG) and smaller (NS) than actual length (to give an idea of bias; we cannot assess bias directly in this case as the actual feature length varies between simulations). As estimators, we consider N8, no. of links N8-1, the scaled N8/0.900 and links/0.900, and the Freeman, Kulpa and corner count methods as well as a stereological estimator.

Here the stereological approach uses a grid of infinitely long, equally spaced parallel lines in the plane, placed at random angle $\theta \in[0, \pi]$ to the horizontal, and such that a reference point on one line is randomly positioned in the plane. 
Given a fixed line spacing to be chosen, the position of all lines in the grid is then fixed, and the number of intersections (if any) of these with the continuous feature is found. Various spacings are used. Unbiased estimation uses the corresponding perpendicular spacing between lines. With a curved image feature, multiple crossings of a single grid line are possible, and with random walk curves the curve can cross itself at a point of intersection with the grid. We avoid this problem here by using polynomial curves. Averaging the results from several different grid angles reduces variability; we averaged results from 10 different angles systematically sampled over the range $[0, \pi]$, i.e. the first angle is drawn from a Uniform distribution over $[0, i n t]$ where $i n t$ is the sampling interval $\pi / 10.0$, and subsequent angles increment each time by int.

\subsection{Angle-based Estimators}

Our other pixel-based methods use estimates of line orientation. Visualizing a line segment of length $l$ and at angle $\theta$, the digitized 8-connected version of Fig. 4(a) has pixels representing no. of columns (Fig. 5), links represent horizontal distance $\mathrm{x}$, and from Fig. 4(a) $l=\frac{x}{\cos \theta}$. The digitized version of Fig. 4(b) has pixels representing no. of rows (Fig. 5), links represent vertical distance y and $l=\frac{y}{\sin \theta}$. Hence we take $\frac{\text { links }}{f(\theta)}$, where $f(\theta)=\cos (\theta)$ if $0 \leq \theta \leq \frac{\pi}{4}$ and $\sin \theta$ for $\theta \in\left(\frac{\pi}{4}, \frac{\pi}{2}\right]$, where $\theta$ is overall angle of the line. These are only applicable for straight lines. Similarly, we can also compute orientation $\theta$ locally at each pixel

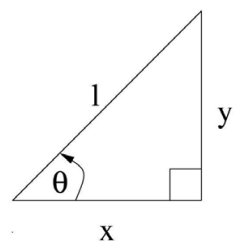

$0<\theta \leqslant \pi / 4$

(a)

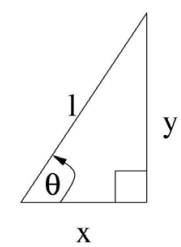

$\pi / 4<\theta<\pi / 2$

(b)

Fig. 4. Visualizing a line segment of length $l$ at angle $\theta$ to the horizontal, the digitized version of (a) has pixels representing no. of columns, while the digitized version of (b) has pixels representing no. of rows

using only a neighbourhood containing one pixel back and one pixel ahead, and add to the estimate $\frac{1}{\cos \theta}$ if $\theta \in\left[0, \frac{\pi}{4}\right]$ or $\frac{1}{\sin \theta}$ if $\theta \in\left(\frac{\pi}{4}, \frac{\pi}{2}\right]$. However this gives too large an answer (c.f. counting pixels rather than links for a horizontal or vertical line; Figs. 5(i) and 5(v)), so we subtract the average of the contributions from the first and last pixels. The local neighbourhood can be enlarged to use $\mathrm{m}$ pixels back and ahead. The window size $\mathrm{m}$ will affect both bias and variance 


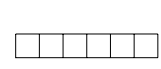

(i) $\theta=0$

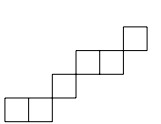

(ii) $0<\theta<\pi / 4$

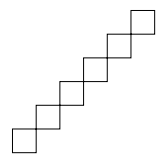

(iii) $\theta=\pi / 4$

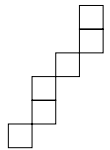

(iv) $\pi / 4<\theta<\pi / 2$

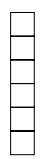

(v) $\theta=\pi / 2$

Fig. 5. Digitized representations of line segments at angle $\theta$ to the horizontal

of the estimator. Optimal size of $m$ will depend on extent of feature curvature. This approach can be applied for straight lines or curves.

\section{Results}

Table 2 shows selected results for the straight lines comparison. Results for the methods using either N8 or no. of links were in each case similar; the best results are shown. N8 and N8-1 underestimate, and are highly variable and inaccurate. Rescaling removes bias and reduces variance, but accuracy is still poor. The Freeman method overestimates but otherwise is superior; Kulpa is noticeably better again and approximately unbiased, and the corner count method better still. However the simple orientation estimators $\mathrm{N} 8 / f(\theta)$ and links $/ f(\theta)$ are very accurate indeed and have low variability, though the former overestimates. The local methods also overestimate but lie between the Kulpa and corner count methods. Corner count is better but not as good as the simple links / $f(\theta)$ which has very low error. The stereological method can be accurate but typically is poor. Averaging the results from several different grid angles improves the results both in terms of MAD and variabilty (as judged by Max AD) but the method is still poorest.

\section{Conclusions}

From this small-scale comparison of pixel-based and stereological estimators of length, so far stereological estimators appear rather poor in comparison to the pixel-based methods. Of the latter, links $/ f(\theta)$ and the corner-count methods are best for straight lines. Using local angles is more useful, and global angles less useful, for curves rather than straight lines, as the angle varies more. Further work is considering digital implementation of stereological estimation rather than using it in the continuous plane. We are also extending this study to projections of 3-D structures.

\section{References}

1. Baddeley, A.: Stochastic Geometry: An introduction and reading-list. International Statistical Review 50 (1982) 179-193 
Table 2. Selected results for the straight line case. Here $f(\theta)=\cos (\theta)$ if $\theta \leq \frac{\pi}{4}$ and $\sin (\theta)$ otherwise; $\theta_{i}$ is a local estimate of angle at pixel $i$. NG and NS are no. of times that the estimate is greater or less than actual length

\begin{tabular}{lccccc}
\multicolumn{1}{l}{ IMAGE SIZE } & $\mathbf{1 2 8}^{2}$ & MAD & Min AD & Max AD NG & NS \\
\hline N8 & 6.84 & 0.0463 & 50.06 & 19 & 81 \\
links $/ 0.900$ & 11.44 & 0.0161 & 36.95 & 64 & 36 \\
\hline & & & & & \\
Freeman & 8.81 & 0.4107 & 11.63 & 100 & 0 \\
Kulpa & 2.85 & 0.0469 & 8.29 & 62 & 38 \\
Corner count & 0.92 & 0.0007 & 2.30 & 58 & 42 \\
\hline & & & & & \\
links $/ \mathrm{f}(\theta)$ & 0.08 & 0.0004 & 0.35 & 64 & 36 \\
$\sum_{i} 1 / f\left(\theta_{i}\right)$ & 2.06 & 0.0168 & 3.94 & 100 & 0 \\
\hline & & & & & \\
Stereological: $\mathrm{h}=2.0$ & 60.66 & 4.477 & 145.6 & 41 & 59 \\
$\mathrm{~h}=10.0$ & 60.38 & 0.3665 & 146.8 & 40 & 60 \\
averaged $(\mathrm{h}=2.0)$ & 32.78 & 1.107 & 69.53 & 28 & 72 \\
\hline
\end{tabular}

2. Buffon, G.L.L. Comte de.: Essai d'Arithmetique Morale. In Supplement a l'Histoire Naturelle, Volume 4 Imprimerie Royale, Paris (1777)

3. Dorst, L. and Smeulders, A.W.M.: Length estimators for digitized contours. Computer Vision, Graphics and Image Processing 40 (1987) 311-333

4. Freeman, H.: Boundary encoding and processing. In B.S. Lipkin and A. Rosenfeld (Eds.), Picture Processing and Psychopictorics. Academic Press, New York (1970) 241-266

5. Freeman, H.: Computer processing of line-drawing images. Computing Surveys 6 (1974) 57-97

6. Glasbey, C.A. and Horgan, G.W.: Image Analysis for the Biological Sciences. Wiley, Chichester (1995)

7. Howard, C.V. and Reed, M.G.: Unbiased Stereology: Three-dimensional Measurement in Microscopy. BIOS Scientific, Oxford (1998)

8. Kulpa, Z.: Area and perimeter measurement of blobs in discrete binary pictures. Computer Graphics and Image Processing 6 (1977) 434-451

9. Vossepoel, A.M. and Smeulders, A.W.M.: Vector code probability and metrication error in the representation of straight lines of finite length. Computer Graphics and Image Processing 20, (1982) 347-64. 